ISSN: 2389-9980 (en línea) | Vol. 47 | No. 108 | Julio-diciembre • 2020 | pp. 102-118

Cuestiones Teológicas | Medellín-Colombia

Como citar este artículo en APA: Bueno Castellanos, Mauricio, De Souza Martins, Marlucio \& Posada-Bernal, Sandra (2020). Espiritualidad y estilos de vida. Cuestiones Teológicas, 47 (108), 102-118. doi: http://doi.org/10.18566/cueteo.v47n108.a06

Fecha de recepción: 30.09.2020 / Fecha de aceptación: 27.10.2020

\title{
ESPIRITUALIDAD Y ESTILOS DE VIDA
}

\author{
Spirituality and Lifestyles \\ Mauricio Bueno Castellanos S.J. ${ }^{\mathrm{I}}$ \\ Marlucio De Souza Martins² \\ SANDra Posada-Bernal ${ }^{3}$ C
}

\section{Resumen}

Las expresiones religiosas y espirituales humanas se pueden relacionar con los estilos de vida de las personas y, en el caso de la educación universitaria, representa un escenario de desarrollo integral de los estudiantes. En este caso, los estudiantes de Teología y miembros de comunidades religiosas realizan procesos de formación que vinculan el cultivo de la espiritualidad cristiana al reconocer y valorar la dimensión no solo corporal, sino también la conciencia espiritual. Este estudio tiene como objetivo caracterizar la espiritualidad y los estilos de vida de los estudiantes de la Facultad de Teología de la Pontificia Universidad Javeriana - Bogotá. Para el desarrollo de esta investigación, se realizó un estudio cuantitativo descriptivo de corte transversal, con una muestra de 34 estudiantes. Se utilizó un cuestionario autoadministrado en Escala Likert, con 30 preguntas, para caracterizar la espiritualidad y los estilos de vida de dichos estudiantes. Los resultados evidencian índices negativos para las categorías: factores nutricionales, actividad física y conducta preventiva. Así mismo, muestran índices positivos para las categorías: prácticas religiosas, prácticas de servicio, orden de valores, relaciones sociales y control del estrés. Se concluye que la espiritualidad tiene una fuerte asociación con los estilos de vida saludables de aquellos que la practican. Se recomienda que, dentro del proceso de formación de los estudiantes de Teología, se incluyan estrategias que promuevan el desarrollo de estilos de vida saludables y que profundicen, disciplinariamente, en las implicaciones que tiene la espiritualidad cristiana y el cuidado de la propia salud.

Palabras clave: Espiritualidad; Estilos de Vida; Educación Universitaria; Estudiantes Universitarios; Espiritualidad Cristiana; Formación Integral; Formación Teológica; Calidad de Vida; Salud Física y Mental; Bienestar.

1 Licenciado en Filosofía y Licenciado en Teología. Magíster en Educación y Magíster en Teología por la Pontificia Universidad Javeriana, Cali, Colombia. Correo electrónico: direccion.pastoral@berchmans.edu.co; mauricio.bueno@ javerianacali.edu.co.

2 Licenciado en Educación Física y Magister en Educación por la Universidad Regional de Blumenau - FURB, Brasil. Estudiante del Programa de Doctorado en Psicología de la Universidad Santo Tomás. Profesor de la Facultad de Educación, Pontificia Universidad Javeriana, Bogotá, Colombia. Correo: mdesouzamartins@javeriana.edu.co

3 Psicóloga por la Universidad de los Andes, Colombia. Magíster en Educación por la Pontificia Universidad Javeriana, Colombia. Estudiante del Programa de Doctorado en Psicología de la Universidad Santo Tomás, Colombia. Correo electrónico: sandraposada@usantotomas.edu.co

1021

Reconocimiento - No Comercia 


\section{ABSTRACT}

Human religious and spiritual expressions could be related to people's lifestyles and, in the case of university education, it represents a scenario of integral development for students. In this case, theology students and members of religious communities carry out formation processes that link the cultivation of Christian spirituality by recognizing and or valuing the dimension not only of the body but also of spiritual awareness. This study aims to characterize the spirituality and lifestyles of the students at the Faculty of Theology of the Pontificia Universidad Javeriana- Bogotá. For the development of this research, a quantitative descriptive study was carried out in a cross section, with a sample of 34 students; a self-administered questionnaire in Likert Scale was used, with 30 questions, to characterize spirituality and lifestyles. The results show negative indices for the categories: nutritional factors, physical activity, and preventive behavior; Likewise, they show positive indices for the categories: religious practices, service practices, order of values, social relations, and stress control. It is concluded that spirituality has a strong association with a healthy lifestyle for those who practice it. It is recommended that strategies that promote the development of healthy lifestyles and that deepen disciplinary knowledge of the implications of Christian spirituality and health care be included within the process of formation of theology students.

Keywords: Spirituality; Lifestyles; University Education; College Students; Christian Spirituality; Integral Formation; Theological Formation; Quality of Life; Physical and Mental Health; Well-Being.

\section{Introducción}

Cada momento histórico trae consigo retos y oportunidades específicos para las diversas generaciones; todo deseo por comprender y dar solución a dichos retos, así como aprovechar las oportunidades, implica el estudio serio y profundo de las relaciones que configuran dicho momento histórico. Una mirada rápida a la situación actual del ser humano permite reconocer una creciente inconformidad con el «status quo»; los avances científicos, económicos y sociales generan más preguntas que respuestas sobre la dignidad, la paz, la salud, la felicidad y el bienestar (Harari, 2015).

Esta realidad cultural ha modificado sustancialmente los modos de trabajar, estudiar y, en general, de desarrollar la vida, a tal punto que pueden llegar a ser perjudiciales para la salud de las personas (Alva y Castillo, 2018). En consecuencia, surge como preocupación mantener un estilo de vida saludable. Al respecto, la Organización Mundial de la Salud (1948) sostiene, en el preámbulo de su Constitución, que: "la salud es un estado de completo bienestar físico, mental y social, y no solamente la ausencia de afecciones o enfermedades" (p.1). Esta definición, si bien está sujeta a cuestionamientos, como el hecho de suponer un consenso universal sobre los términos que la componen (Alcántara, 2008), también es cierto que sirve de base para el cumplimiento de las competencias de la OMS, que es el máximo organismo internacional en materia de salud.

Esta definición permite comprender que el concepto salud no hace referencia exclusivamente al ámbito corporal, sino que se refiere a la interacción de diversos factores, tal como lo afirma el profesor Briseño-León, citado por Alcántara (2008): “[la salud] es la síntesis de una multiplicidad de procesos, de 
lo que acontece con la biología del cuerpo, con el ambiente que nos rodea, con las relaciones sociales, con la política y la economía internacional” (p. 15). Por consiguiente, la atención que hoy exige la salud y el cultivo de estilos de vida saludable, implican una mirada integral del ser humano.

Palacios (2015) señala que el presente momento histórico: "reclama un sujeto dispuesto a comprender el orden del mundo sin perderse en él" (p. 461); recogiendo las palabras de Bauman (2013), un "hombre capaz de enfrentar la tensión entre la fluidez de la modernidad y la construcción de identidad” (p. 22). Frente a este reto, la espiritualidad emerge como una dimensión humana. La Asociación de Colegios Jesuitas de Colombia(ACODESI), afirma que la espiritualidad permite "comprender el mundo y dar sentido a los acontecimientos, procurando la construcción individual e integral del ser humano" (2003, p. 48). El teólogo José María Castillo (2008) señala cómo, en la actualidad, más personas buscan en la espiritualidad espacios de integración y coherencia ante la realidad

Cada día aumenta el número de personas que experimentan más y más, no ya la simple curiosidad por el esoterismo o cosas parecidas, sino la necesidad de vivir una espiritualidad coherente con las nuevas situaciones debidas al rápido y profundo cambio cultural del momento presente. (p. 17)

La espiritualidad, y particularmente la cristiana, se entiende como una dimensión humana, entre otras muchas, la cual permite "comprender el mundo y dar sentido a los acontecimientos, procurando la construcción individual e integral del ser humano" (ACODESI, 2003, p. 48).

La relación entre espiritualidad y salud no es nueva. En los últimos años se han elaborado diversos estudios que muestran el interés por ahondar en esta interacción, ya sea desde las categorías espiritualidad y su relación con la dinámica social (Krmpotic, 2016), espiritualidad y cuidado de la salud (Uresti, et al., 2011; Navas y Villegas, 2006), espiritualidad y desarrollo humano (Palacio, 2015) o, los más recientes, espiritualidad y estilos de vida saludables (Alva, 2018; Quispe, 2018). Estos trabajos son una breve muestra de la producción investigativa, lo cual sugiere el amplio espectro que existe en materia de estudio. De acuerdo con Salgado (2015), si bien la relación entre espiritualidad y salud fueron desconocidas por los estudios académicos en los siglos pasados, actualmente existe un cambio relevante: "la espiritualidad ha sido reconocida como parte integral de la salud, el bienestar y la calidad de vida" (Sánchez, 2008, p. 92); con lo cual, no sólo se evidencia su relación, sino que se ratifica el aporte que otorga la espiritualidad al cultivo de la salud física y mental.

Siguiendo el trabajo de Cornejo y Martín (2019), la noción espiritualidad ha presentado un giro hacia una espiritualidad integrada, fundamentada en tres grandes procesos: la evolución de la espiritualidad cristiana hacia una espiritualidad individual con el desarrollo de las expresiones corporales del religioso; la espiritualidad se ha abierto un espacio relevante en la literatura médica como dimensión clínica; y, la psicología humanista, junto con algunas disciplinas afines, ha profundizado en la relación espiritualidad y salud/bienestar. Frente a este amplio campo de indagación, la presente investigación se focalizó en la pregunta: ¿qué relación existe entre la vivencia de la espiritualidad cristiana y el cultivo de estilos de vida saludables?

Por espiritualidad entendemos una "dimensión fundamental del ser humano la cual se encuentra en estrecha relación con las demás dimensiones” (Arango y Solano, 2016, p. 124); Tamayo (2007) la 
plantea como "la corporalidad o la comunicación; lo cual implica el reconocimiento de su mutuo influjo y afectación” (p.1). El crecimiento de alguna de las dimensiones del ser humano comporta algún tipo de afectación en las demás, de lo contrario, estaríamos ante una fragmentación del ser humano, o un falso crecimiento (Silva, 2019). La espiritualidad, para Salgado (2015), "implica el modo como los seres humanos nos relacionamos con nosotros mismos, con los demás y con Dios, e implica la manera de construir sentido a la vida" (p.92). Por otra parte, Hadot (2006) menciona que la: "espiritualidad, y el ejercicio de ella, implica el desarrollo humano de autoconciencia sobre lo que ocurre en su interior y el modo como se relaciona con la exterioridad" (p. 23).

Al hablar de espiritualidad cristiana, nos referimos, explícitamente, a la experiencia que la comunidad posee de la persona de Cristo, lo que San Pablo llama: "La vida según el Espíritu" (Rom 8, 2-17). Siguiendo a González (2019), la espiritualidad cristiana es un: "estilo de vida guiado por el espíritu de Cristo" (p. 339), y Cristo, en tanto que presencia de Dios en la historia, asume la humanidad integralmente. La espiritualidad cristiana se preocupa de la vida humana en su integralidad, tal como lo señala Pablo VI en la Gaudium et spes (1967):

Los gozos y las esperanzas, las tristezas y las angustias de los hombres de nuestro tiempo, sobre todo de los pobres y de cuantos sufren, son a la vez gozos y esperanzas, tristezas y angustias de los discípulos de Cristo. Nada hay verdaderamente humano que no encuentre eco en su corazón. (No. 1)

La teóloga Mónica Ukaski (2018) seńala que la vida espiritual se define como un vivir desde la experiencia de Dios, que implica la vida desde el yo profundo. Grüm, citado por Ukaski (2008), menciona que: "vivir de acuerdo con nuestro verdadero yo, significa vivir según la propia naturaleza, sin eliminar ningún sector de la vida, de modo que a través de todo lo que hay en nosotros "Dios sea glorificado"” (p. 639).

Hablar de estilos de vida saludables hace referencia a las acciones rutinarias producto de un conocimiento originado en las estructuras sociales (Bourdieu, 1998). Siguiendo a Nahas (2000), estas acciones habituales reflejan las actitudes, los valores y las oportunidades en la vida, las cuales pueden estar clasificadas por factores negativos o positivos que manifiestan un estado de salud o bienestar. De acuerdo con Alva y Castillo (2018), un estilo de vida saludable se asume como el: "conjunto de comportamientos y hábitos adoptados que han desarrollado las personas, cuyos valores pueden beneficiar la salud y también lo contrario" (p. 31).

La espiritualidad cristiana ha tenido una estrecha relación con los estilos de vida, pues su propuesta de ser humano implica la encarnación de la experiencia divina en el mundo de la vida, lo cual encuentra su culmen en la persona de Jesús. Ahora bien, en la actualidad surge la pregunta, ¿Qué tipo de relación se establece entre la espiritualidad cristiana y los estilos de vida de quienes la practican?, pues no resulta evidente el modo como esta se encarna en la vida concreta de los seres humanos de hoy.

$\mathrm{Al}$ respecto, vale la pena recordar el texto escrito por Alberto Hurtado, S.J., ¿Es Chile un país católico? (1941), donde el autor cuestiona la premisa de una catolicidad vivida desde la espiritualidad cristiana, porque encuentra que la sociedad chilena actúa de manera contraria respecto de los principios que ella propone. En parte, algunas tendencias del catolicismo en Latinoamérica han transformado la fe cristiana en un discurso con un marcado acento institucional y doctrinal, más que experiencial o vivencial, lo cual 
ha producido la afirmación de ciertas características dualistas de la espiritualidad. Un claro ejemplo de ello puede confirmarse en el artículo publicado en el periódico El Tiempo, en la sección Redacción Vida (2017), donde se afirma que: "Colombia es un país de creyentes, mayoritariamente católicos, pero en general poco comprometidos con su fe" (El Tiempo).

Esta realidad contrasta con una visión unitaria de Dios, el mundo y el ser humano sostenida por el cristianismo. Durante sus primeros siglos fue fundamental definir cuál es la comprensión de ser humano que subyace a la experiencia cristiana. Esta pregunta surge por los diálogos que vivieron los seguidores de Cristo con otras corrientes de pensamiento presentes en la cultura greco-romana de los primeros siglos, tales como el gnosticismo, el arrianismo o maniqueísmo, entre otras. Fruto de estas relaciones, el cristianismo naciente logró constituir una antropología optimista, a diferencia de las pretensiones gnósticas del momento. El optimismo frente al ser humano obedeció, en gran medida, a una lectura de la encarnación desde el esquema de la asunción: Dios asume la humanidad, en todas sus dimensiones, en la persona de Cristo, permitiendo entender la integridad del ser humano como criatura de Dios. Al respecto, Dussel (1974) señala que: "Las estructuras de la antropología cristiana se originaron en el fértil suelo del mundo judío de Palestina, y posteriormente de Antioquía y Alejandría... El hombre es considerado una creatura divina, constituido en el ser desde su origen radical e individualmente" (p. 58).

Proponer esta antropología desde la espiritualidad cristiana permite concluir que toda experiencia de esta espiritualidad implica, a su vez, una experiencia de cuidado del ser humano en todas sus dimensiones. En esta línea de ideas, el presente texto se enfoca en analizar la relación que existe entre una experiencia concreta de la espiritualidad cristiana y el cuidado de la dimensión corporal. Al respecto, Grüm y Dufner (2000) distinguen entre una espiritualidad cristiana desde abajo y una espiritualidad cristiana desde arriba, perfilando la espiritualidad desde abajo como una dimensión de la espiritualidad que reconoce los datos espaciotemporales, como el lugar en el que acontece la pregunta por Dios: "En la espiritualidad desde abajo se trata sobre todo de conseguir abrirse a las relaciones personales con Dios en el punto preciso en que se agotan y cierran todas las posibilidades humanas" (p. 2).

Esta investigación se desarrolló con estudiantes de la Facultad de Teología de la Pontificia Universidad Javeriana - Bogotá, teniendo presente que la mayoría de dichos estudiantes participan en procesos formativos dentro de la comunidad cristiana, es decir, profesan abiertamente su vínculo con la espiritualidad cristiana. El interés de esta investigación por esta población, en particular, surge, en gran medida, de la invitación que realiza el Papa Francisco (2018) en su constitución apostólica Veritatis Gaudium, donde señala la "necesidad de una auténtica hermenéutica evangélica para comprender mejor la vida, el mundo, los hombres, no de una síntesis sino de una atmósfera espiritual de búsqueda y certeza basada en las verdades de razón y de fe" (No. 3). En la Veritatis Gaudium, Francisco invita a las universidades, facultades e institutos eclesiásticos a generar nuevos espacios de investigación:

en los que estudiosos procedentes de diversas convicciones religiosas y de diferentes competencias científicas puedan $[\ldots]$ entrar en un diálogo entre ellas orientado al cuidado de la naturaleza, a la defensa de los pobres, a la construcción de redes de respeto y fraternidad. (No.5)

Así mismo, entendemos que uno de los fines fundamentales de la educación es la formación integral de los individuos, lo cual implica el desarrollo de hábitos de vida saludable; por ello, hacemos eco del 
informe de la UNESCO La educación encierra un tesoro. Delors (1996) señala claramente, que los pilares de la educación no son solo aprender a conocer y aprender a hacer, sino además, aprender a vivir juntos y aprender a ser.

Fruto de esta apuesta investigativa, el presente trabajo se trazó como objetivo caracterizar la espiritualidad y los estilos de vida de los estudiantes de la Facultad de Teología de la Pontificia Universidad Javeriana- Bogotá. Para desarrollar este objetivo se presentará la metodología utilizada en la investigación. Seguidamente se darán los resultados, discutiendo cada elemento en la medida en que se evidencie. Finalmente, se plantearán las conclusiones.

\section{Metodología}

El enfoque metodológico utilizado para esta investigación está centrado en un estudio cuantitativo descriptivo de corte transversal para caracterizar los estilos de vida de los estudiantes de la Facultad de Teología de la Pontificia Universidad Javeriana- Bogotá, Colombia. De acuerdo con Hernández et al. (2014) y Hernández y Mendoza (2018), este tipo de metodología busca especificar las particularidades y aspectos importantes de cualquier fenómeno analizado y describir las tendencias de un grupo o población. En este sentido, el enfoque metodológico cuantitativo utilizado en este estudio se justifica por medio de la recolección de datos primarios de los estudiantes de Teología y su relación con el entorno, ya que dichos estudiantes pertenecen a procesos formativos dentro de la comunidad cristiana y profesan abiertamente su vínculo con la espiritualidad cristiana.

Por lo anterior, basándonos en las teorías de Bronfenbrenner (1987) y Shelton (2019), el entorno incide en la forma en que la persona se desarrolla e interactúa dentro de un sistema social y cultural, lo que conforma un estilo de vida. A su vez, las principales investigaciones sobre estilos de vida están centradas, en su mayoría, en un estudio cuantitativo a partir de cuestionarios con escalas de actitudes y de valores tipo Likert, cuya finalidad es verificar las conductas cambiantes que tiene una "persona dentro del ambiente y su capacidad para descubrir, mantener o modificar las propiedades del ambiente en que se encuentra" (Espinoza y Carpio, 2015, p. 118).

A su vez, este estudio se caracteriza como descriptivo, debido que se realizó la recolección de datos sobre diferentes aspectos de los estilos de vida y de la espiritualidad de la población. De acuerdo con Hernández et al. (2014) y Hernández y Mendoza (2018), en un estudio descriptivo se miden, de manera más independiente, los conceptos y variables para mostrar, con precisión, los ángulos o dimensiones de un fenómeno o situación. De igual modo, es una investigación que analiza datos y variables recopiladas en un período de tiempo sobre una determinada población, por lo cual se denomina estudio de corte transversal.

Para la realización del estudio, se seleccionó una muestra de 34 estudiantes de pregrado pertenecientes a la Facultad de Teología de la Pontificia Universidad Javeriana - Bogotá, que cumplieron con los criterios de inclusión: a) estudiantes matriculados en los cursos: Religiones y Ecumenismo, que tiene como objetivo 
identificar las respuestas que diversas religiones y confesiones cristianas han dado a los grandes interrogantes del ser humano con respecto a la realidad y a su existencia, para enriquecer su propia comprensión dentro de una fe responsable; Teología de la Acción III, compuesta por talleres formativos en que los estudiantes logran, desde la práctica concreta, apropiar significativamente los postulados epistemológicos y procedimentales de la teología de la acción educativa; b) mayores de 18 años; c) estudiantes que están de acuerdo con su participación en la investigación y que hayan realizado la lectura y firma del consentimiento informado.

La muestra presenta una edad promedio del $38,5 \pm 19,5$ años, que para el género femenino representa el 23,5\% ( $\mathrm{n}=8)$ y el masculino el 76,5\% ( $\mathrm{n}=26)$. De acuerdo con López-Roldán (2017), para obtener la validez de una muestra representativa en poblaciones finitas se hace necesario trabajar con el mínimo dentro de un margen de error del 5\% y un nivel de confianza del 95\%. En este sentido, de los 37 estudiantes que hacían parte de los dos cursos, solo 34 cumplieron con los criterios de inclusión, con lo cual, la cantidad de estudiantes que participaron supera la validación de la muestra del 95\% $(\mathrm{p}<0.05)$.

A su vez, se justifica la selección de la muestra (estudiantes universitarios) a partir de los estudios de Erikson (1998), Papalia et al. (2012), Delgado (2015) y Harris (2019), donde se afirma que los jóvenes universitarios presentan cambios significativos de conductas, ya que existe una adaptación y transición al nuevo entorno que, debido a las exigencias del nuevo contexto, pueden causar un incremento en los cambios de estilos de vida.

Por otro lado, según De Souza et al. (2017): "no hay un método estándar diseñado para medir la prevalencia de una exposición o resultado en una población definida y en un punto específico de tiempo" (p. 234). Siendo así, dentro de los varios instrumentos existentes para evaluar el estilo de vida de las personas, se optó por utilizar un formulario estandarizado propuesto por Nahas (2013), que presenta una escala Likert, muy común en la investigación social para medir las actitudes y conocer el grado de conformidad de la muestra (Fernández, 2005).

En este sentido, se utilizó el cuestionario del Pentágono del Bienestar (Nahas, 2013), que presenta un modelo de instrumento autoadministrado para evaluar los componentes del estilo de vida que pueden afectar la salud y el bienestar de las personas. Los componentes evaluados incluyen quince preguntas divididas en cinco criterios de análisis: 1) factores nutricionales; 2) actividad física; 3) conducta preventiva; 4) relaciones sociales; 5) control del estrés. Para caracterizar la espiritualidad de los estudiantes de Teología, se estructuró un cuestionario con quince preguntas divididas en tres criterios de análisis: 1) prácticas religiosas; 2) prácticas de servicio; y 3 ) orden de valores.

Estas categorías presentan los estilos de vida de los estudiantes de la facultad de Teología, de tal manera que pudiese visibilizarse la relación que se entabla con la experiencia de la espiritualidad cristiana. Dichas categorías no pretenden agotar la experiencia de la espiritualidad cristiana, ya que, a lo largo de la historia del cristianismo, han existido innumerables expresiones y concreciones históricas de la espiritualidad. Para el presente estudio conviene acotar la experiencia de la espiritualidad cristiana en estas tres categorías dirigidas a analizar comportamientos de los individuos en su dimensión personal, de relación con otros y de valoración vital. 
Como procedimiento para la aplicación de los instrumentos, se obtuvieron los permisos institucionales por parte de la Facultad de Teología. A su vez, se compartió, por correo electrónico, la invitación a los estudiantes para participar de la investigación, contestando un cuestionario en línea a través del uso de la herramienta tecnológica Google Formularios. El correo contenía un enlace a GF para el desarrollo de la encuesta, además de un apartado con el consentimiento informado y la explicación de los objetivos de la investigación.

Por lo anterior, las consideraciones éticas del estudio se realizaron siguiendo las normas deontológicas reconocidas por la declaración de Helsinki y el Código de Ética de la Asociación Americana de Investigación Educativa (AERA, 2011). Así, en conformidad con la Resolución No. 8430 de 1993 del Ministerio de Salud y Protección Social (2013), la participación en este estudio es considerada como de riesgo mínimo. A su vez, en cumplimiento con lo previsto en el Decreto 1377 de 2013 del Ministerio de Comercio, Industria y Turismo (2013), reglamentario de la Ley 1581 de 2012 (Congreso de Colombia, 2012), se solicitó la autorización para que, de manera previa, libre, expresa y debidamente informada, la persona permita dar tratamiento a los datos personales de identificación, contacto, socio económicos, académicos, suministrados en esta investigación. Además, los participantes fueron informados de que los datos personales serían tratados de acuerdo con los principios de acceso y circulación restringida, seguridad, y confidencialidad.

Por último, para la organización, análisis e interpretación de los datos, se codificaron las variables a través de una rejilla de sistematización de la información, conforme las recomendaciones de Little (2013a; 2013b). Para el análisis estadístico descriptivo se utilizó el programa IBM SPSS Statistics V25.

\section{Resultados y discusión}

A continuación, se presentan los resultados más significativos en la investigación, de acuerdo con cada una de las categorías trabajadas. Se iniciará por los estilos de vida y luego se abordará la espiritualidad.

\section{Factores nutricionales}

Para esta dimensión de los estilos de vida, se evidenció un índice negativo en el ámbito de los factores nutricionales, pues el $56 \%(n=19)$ no incluye frutas y verduras en su dieta diaria, con una tendencia mayor para el género masculino. A su vez, la muestra presenta índices positivos en algunos ámbitos nutricionales, donde el 65\% ( $n=22)$ evita consumir alimentos grasosos y azucarados. Además, el 70\% ( $n=24)$ consumen entre 4 a 5 porciones de alimentos al día. Esta tendencia positiva presenta una tendencia similar entre los géneros (ver figura 1).

En esa misma línea, los resultados presentan una relación muy similar con investigaciones realizadas por De Souza Martins et al. (2017), a partir de un estudio sobre los estilos de vida en estudiantes universitarios, con una muestra de 1410 estudiantes con edades entre 24 y 29 años. Se evidenció un índice negativo en los estilos de vida, específicamente en los factores nutricionales, donde: "el $61 \%$ no incluye frutas ni verduras en su dieta diaria; el 57\% no evita el consumo de alimentos ricos en grasa, y el 25\% de la muestra consume menos de cinco alimentos durante el día” (p. 236). El estudio concluyó que existe una tendencia de estilos de vida no saludables que puede afectar negativamente la salud de esta población. Otro estudio, realizado 
Figura 1. Factores Nutricionales

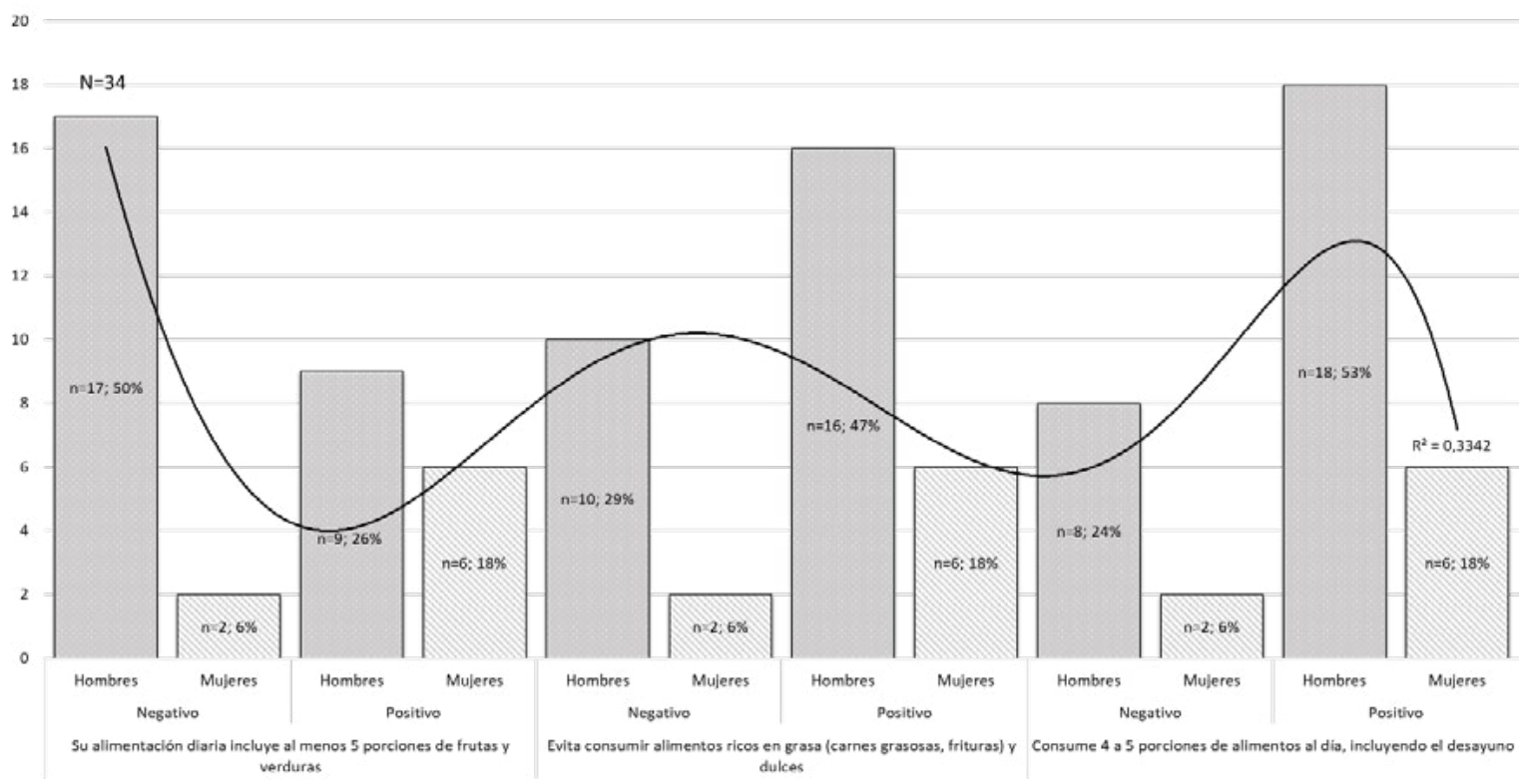

Fuente: Elaboración propia (2020).

por De Souza et al. (2015), evidenció resultados similares, lo que demuestra una creciente preocupación, entre estudiantes universitarios, por un estilo de vida asociado con la calidad de vida saludable.

Por otro lado, recientemente se ha encontrado que los estudiantes están presentando estilos de vida que afectan su estado de salud, los cuales han sido adquiridos en etapas como la adolescencia, tendiendo a persistir hasta la edad adulta (Zea et al., 2014). Quispe (2018), en su investigación, señala que, si bien: "encontró que el grado de calidad de la práctica religiosa y grado de calidad del estilo de vida, tienen una relación positiva y significativa” (p. 82), en la investigación realizada no se encontró una relación significativa entre espiritualidad cristiana y estilos de vida saludables.

Esta evidencia exige estudios específicos para identificar datos más concluyentes, razón por la cual se plantea, desde ya, la necesidad de posteriores investigaciones en esta línea. En palabras de Quispe (2018): "Se podría requerir otras disciplinas de la espiritualidad cristiana para que el nivel del estilo de vida se eleve” (p. 83).

\section{Actividad física y conducta preventiva}

En el ámbito de la actividad física, se presenta un índice negativo, donde el 68\% (n=23) de la muestra no realiza al menos 30 minutos de actividad física moderada o intensa de forma continua (sin descanso 
en este intervalo de tiempo). Igualmente, el $59 \%(\mathrm{n}=20)$ no realiza ejercicios que involucran fuerza y estiramiento muscular al menos dos veces por semana con predominancia del género masculino para ambos casos. Para la conducta preventiva, hay una incidencia negativa, ya que el $47 \%(n=16)$ raramente conoce su presión arterial y su nivel de colesterol. Nuevamente, el género masculino tiende a no realizar los controles regulares de su estado clínico.

Figura 2. Actividad Física y Conducta Preventiva

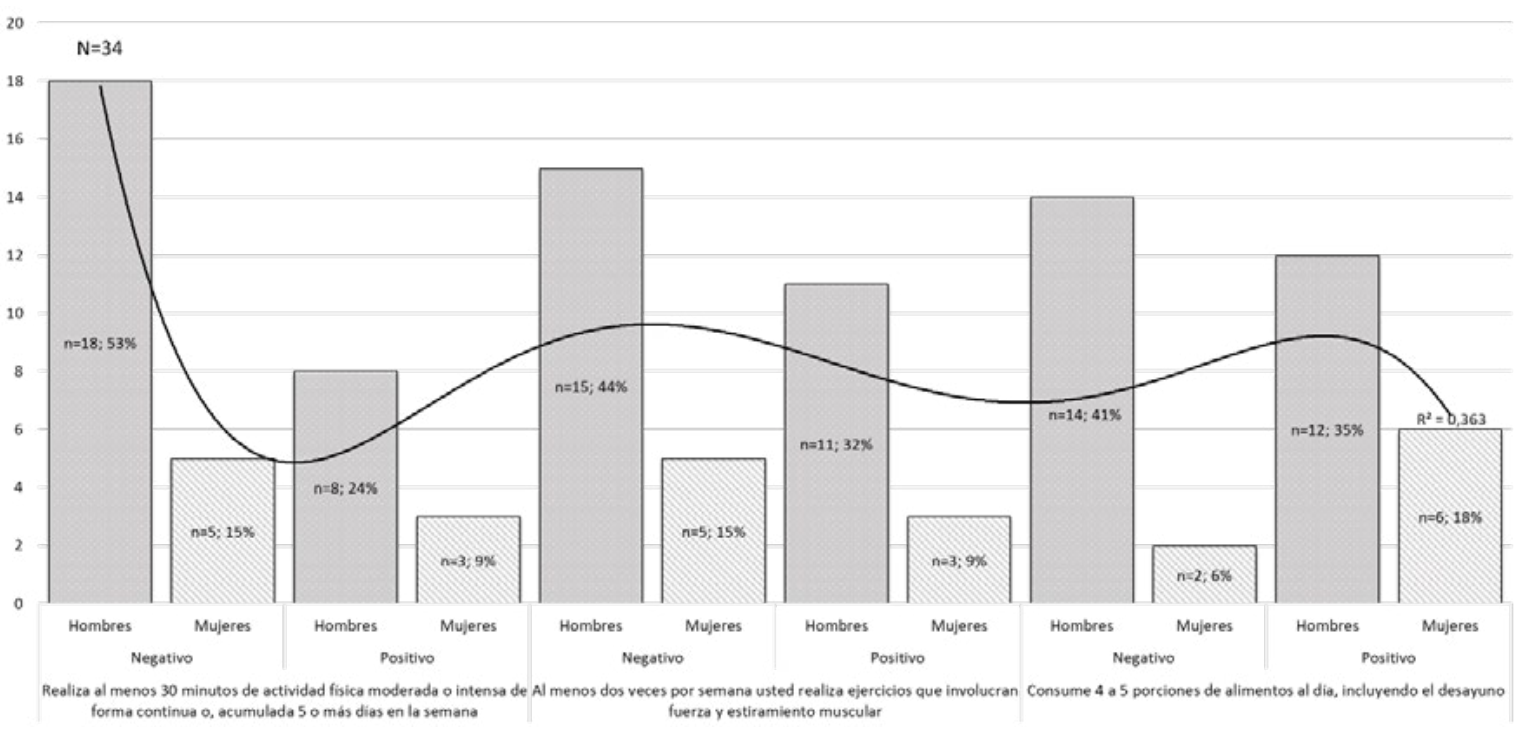

Fuente: Elaboración propia (2020).

En la literatura se evidencian resultados similares. Para Gómez-Acosta (2018), los estilos de vida son concebidos como un conjunto de conductas que determinan el proceso de salud-enfermedad en combinación con la vulnerabilidad biológica, la reactividad psicofisiológica, la edad y el sexo. Del mismo modo, Da Costa et al. (2019), han mostrado que solamente el 12\% de los estudiantes dedica tiempo para realizar actividad física moderada-a-vigorosa y $41 \%$ actividad física ligera. Así, el restante $47 \%$ se emplea en actividades que no implican movimiento. En este sentido, Moscoso (2013) afirma que el 89\% de los estudiantes universitarios presenta hábitos de alimentación deficiente y el 46\% realiza actividad física baja, lo que genera una predisposición al desarrollo de las Enfermedades Crónicas No Transmisibles (ECNT).

Este índice negativo en los factores de actividad física vuelve a poner sobre el papel la necesidad de estudios ulteriores, con el fin de determinar, con una mayor exactitud, la relación entre espiritualidad cristiana y actividad física. Esta falencia surge al reconocer en la espiritualidad cristiana, como ya se ha planteado previamente, el interés por el cuidado y el cultivo de la corporalidad, y la actividad física es una variable fundamental de dicho cuidado. 


\section{Relaciones sociales y control del estrés}

A su vez, para las variables de relaciones sociales y control del estrés, la muestra se distribuye dentro del índice positivo, conforme demuestra la Figura 3.

Figura 3. Relaciones Sociales y Control del Estrés

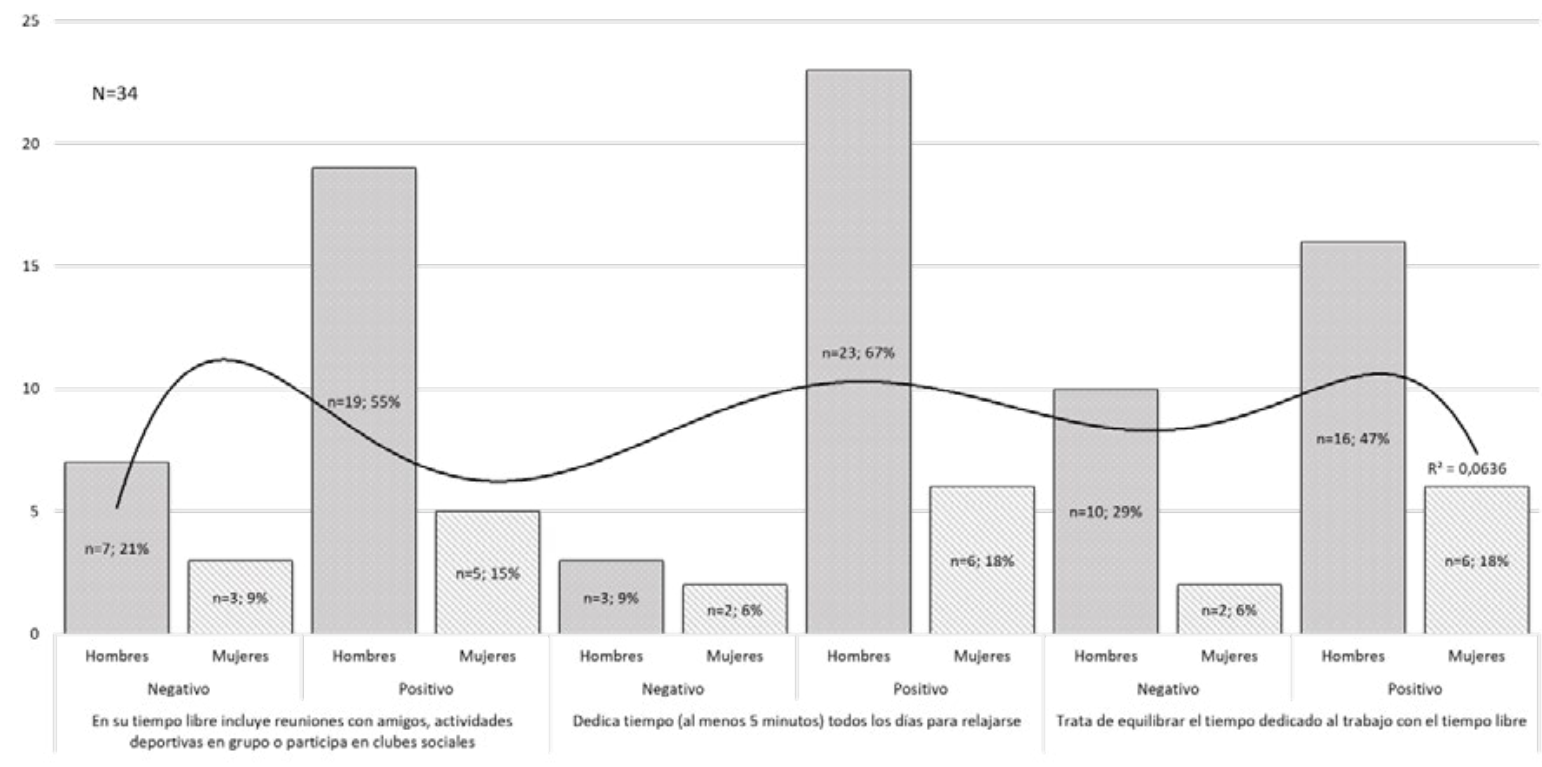

Fuente: Elaboración propia (2020).

Las relaciones sociales y el control del estrés son considerados determinantes psicológicos en la salud mental de las personas, que incluyen no solo las características individuales para gestionar pensamientos, emociones, comportamientos e interacciones con los demás, sino también factores sociales, culturales, económicos, políticos y ambientales (OMS, 2013). Además, para Gómez-Acosta (2018): "los factores psicológicos pueden predecir tanto la salud mental como el estilo de vida” de las personas” (p. 161).

Los resultados hallados por el estudio presentan datos distintos de los encontrados en la literatura. En un estudio realizado en Brasil sobre la insatisfacción de los estudiantes con la imagen corporal y su relación entre el estrés, la autoestima y los problemas alimentarios, se evidenció que, de los 238 estudiantes que participaron del estudio, las mujeres presentaron un mayor riesgo para desórdenes alimenticios, mayor preocupación con la imagen corporal, más estrés y una menor autoestima en relación con los hombres. A su vez, el estudio muestra una relación positiva entre la imagen corporal con el comportamiento alimentario de la población, presentando factores de bulimia en una población tan joven (Da Silva et al., 2018). 
La diferencia hallada entre los datos positivos del presente estudio y los datos negativos que la literatura muestra puede explicarse al indicar que la población de estudio pertenece a procesos formativos con un fuerte acento espiritual cristiano, el cual incorpora el trabajo explícito de las relaciones sociales, así como espacios de oración, individual y colectiva, e interiorización, los cuales, de acuerdo con Quiceno y Vinaccia (2009): "proveen al individuo el crecimiento espiritual continuo, apoyo psicológico, propósito en la vida e interacción social” (p. 324).

Conviene señalar que, si bien los resultados encontrados en relaciones sociales y control del estrés son contrarios a la literatura sobre estudiantes universitarios, en lo relativo a la relación espiritualidad y estilos de vida saludables, se confirma su mutua incidencia: a mayor espiritualidad, mayores relaciones sociales y mayor control del estrés (Navas y Villegas, 2006; Barrero et al., 2001; Rodriguez, 2018; Cornejo, et al., 2019). Al respecto, Krmpotic (2016) señala que el desarrollo de la espiritualidad conlleva al sano desarrollo de la salud y los servicios sociales; así mismo, Koening (2013) afirma que, en los hallazgos de las investigaciones realizadas, las personas encuentran, en la espiritualidad, confort ante la ansiedad y la desesperación.Dice: "resultan menos depresivos y ansiosos, y cuando la religión se incorpora en los tratamientos tradicionales para ansiedad y depresión los resultados son mejores” (p. 71).

\section{Espiritualidad}

Además, para la dimensión de la espiritualidad, se evidencian indicadores positivos en todas las categorías, sin distinción del género. Para prácticas religiosas, al $91 \%(\mathrm{n}=31)$ la vida espiritual les ha brindado elementos para un mayor y mejor conocimiento de sí; además, el $82 \%(\mathrm{n}=27)$ afirma que sus prácticas religiosas aportan elementos para atender su salud física. En las prácticas de servicio, el $88 \%(\mathrm{n}=30)$ ha construido amistades profundas y permanentes en los grupos humanos con los cuales comparte; también, el 94\% $(\mathrm{n}=32)$ reconoce y atiende las necesidades de las personas con quienes comparte. Por último, en el orden de valores, el $88 \%(\mathrm{n}=30)$, dentro de su actividad pastoral, se interesa por el bienestar físico y corporal de las personas con quienes interactúa; a su vez, el $97 \%(n=33)$ comparte gratuitamente su tiempo con personas que no pueden retribuirle (ver figura 4).

Una relación importante en esta investigación es el desarrollo de la espiritualidad y los estudios de Teología, planteado desde el inicio, al seleccionar la muestra de estudiantes de entre aquellos pertenecientes a la facultad de Teología. Al respecto, conviene recordar documentos magisteriales como el decreto Optatam Totius del Vaticano II (1965), donde se recuerda la necesidad de adecuar la formación teológica a la vida espiritual: "La formación espiritual ha de estar en íntima conexión con la intelectual y pastoral y ha de darse sobre todo con la ayuda del director espiritual" (No 8). Si bien este documento se escribe en el horizonte de la formación sacerdotal, de acuerdo con Ascua (2011), la relación entre la formación teológica y la espiritualidad hace parte de la vocación cristiana, no solamente de un grupo particular. Azcuy (2011) plantea que: "de hecho, de manera semejante a cómo se hace referencia en la formación sacerdotal, la noción de espiritualidad también aparece vinculada a los institutos de vida religiosa (cf. PC 6b) y a la vida de los laicos (cf. AA 4)” (p. 260). 
Figura 4. Dimensión de la Espiritualidad

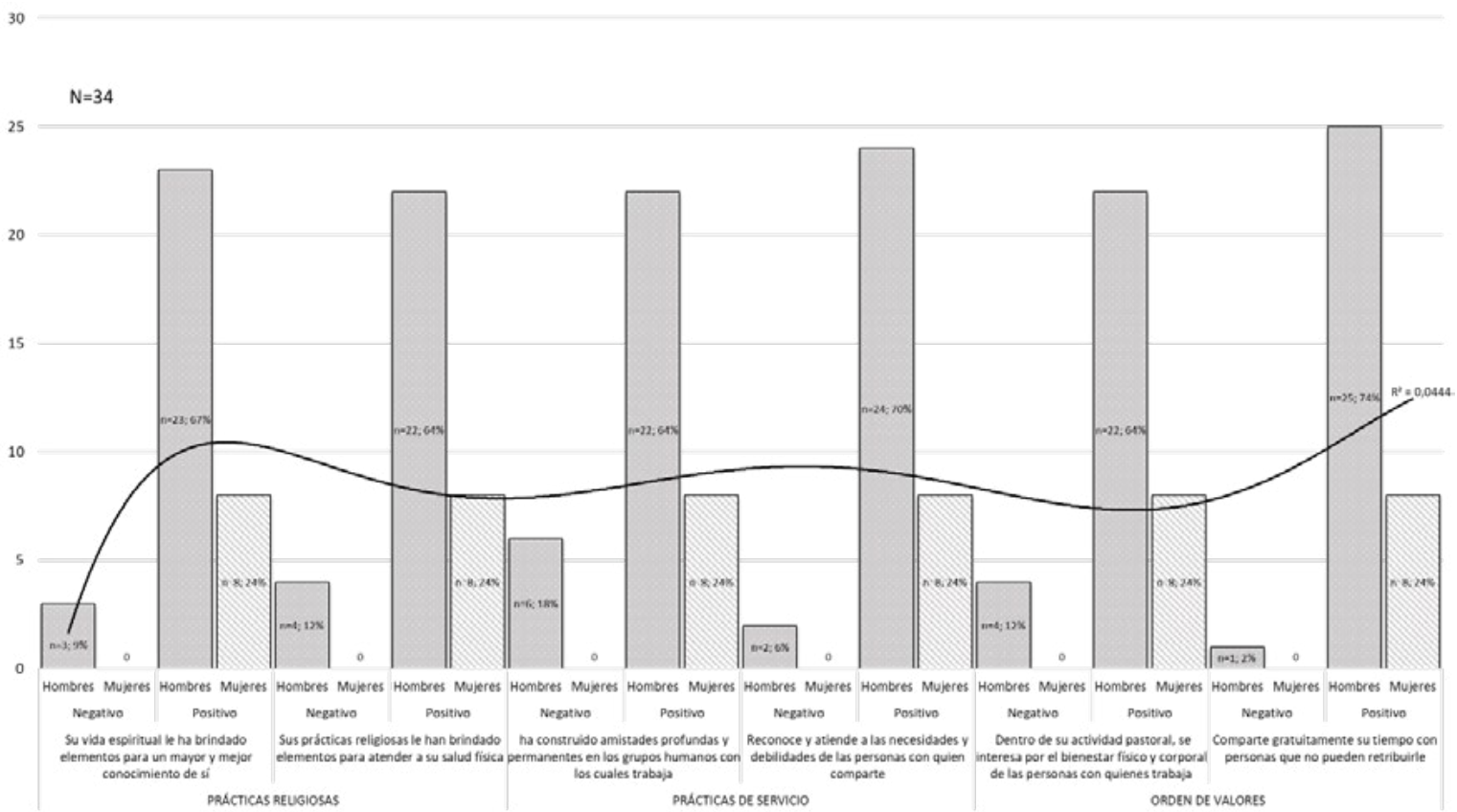

Fuente: Elaboración propia (2020).

En esta línea de ideas, el Papa Francisco (2018) señala que el primer criterio para la "renovación sabia y valiente" (No 3) de los estudios eclesiásticos (en este caso, el estudio de la Teología) implica la contemplación y la introducción espiritual, intelectual y existencial en el corazón del kerigma. La centralidad del kerigma posiciona la espiritualidad en el horizonte de la encarnación, con lo cual se ratifica nuevamente la vocación integral que tiene el ser humano.

\section{Conclusiones}

La literatura revisada ha determinado que la espiritualidad está asociada estrechamente con estilos de vida saludables de quienes la practican. La relación entre los estilos de vida saludables y la práctica de la espiritualidad cristiana, en un grupo humano en formación explícita dentro de la religión cristiana, resulta un tema de interés, tanto para los sujetos que intervienen en el estudio como para el interés mismo de la teología, que vuelve la vista sobre la población que experimenta los procesos formativos. La muestra evidencia una tendencia no saludable en los estilos de vida, pero una tendencia positiva para las variables de la espiritualidad. Lo anterior abre la pregunta sobre los procesos de reflexión e introspección que la población estudiada realiza respecto de la materia de estudio. 
$\mathrm{Al}$ respecto, puede verse una relación con las investigaciones realizadas en poblaciones de estudiantes universitarios, tales como Quispe (2018), Alva y Castillo (2017), De Souza et al., (2015) y (2017), Zea et al., (2014). Estos académicos señalan la necesidad que existe en los estudios universitarios de incorporar la reflexión sobre la espiritualidad y su injerencia directa en los hábitos de vida de la población estudiantil. Al hablar de la formación en espiritualidad y estilos de vida saludables en estudiantes en psicología, Salgado (2015) concluye la necesidad de evaluar las mallas curriculares de las universidades para identificar posibles vacíos en la formación, con el finde implementar medidas que corrijan las deficiencias existentes.

En la misma línea, Coral y Vargas (2014) afirman que la universidad debe procurar espacios que fomenten explícitamente estilos de vida saludables. Lucheti et al. (2012), al investigar estudiantes de escuelas médicas en Brasil, señalan que, si bien existen algunos cursos en las universidades que promueven estilos de vida saludables: "desafortunadamente, la mayor parte de la enseñanza sobre S / $\mathrm{H}$ [estilos saludables] no está estandarizada y pocas escuelas incluyen la oportunidad de que los estudiantes practiquen realmente lo que aprenden" (p. 8). Esta realidad, percibida en estudiantes de ciencias de la salud, puede aplicarse, mutatis mutandi, a estudiantes de Teología, puesto que lo que aprenden en la disciplina revierte necesariamente en un comportamiento directo con el cuidado o no de la propia salud.

En este sentido, se recomienda que, dentro del proceso de formación de los estudiantes de Teología, se incluyan estrategias que favorezcan la concientización sobre la importancia de los factores nutricionales, de la actividad física y de conductas preventivas para llevar una mejor calidad de vida, así como profundizar disciplinariamente en las implicaciones de la espiritualidad cristiana y el cuidado de la propia salud.

Esta investigación presenta algunas limitaciones que deben ser tomadas en cuenta para futuros trabajos. Dado que no existen muchos estudios que articulen la espiritualidad con los estilos de vida, se hace necesario continuar con investigaciones que incluyan una muestra más amplia con la finalidad de tener un panorama del fenómeno y datos más concluyentes.

\section{Referencias}

Asociación de Colegios Jesuitas de Colombia (1986). Caracteristicas de la Educación de la Compañia de Jesús. Bogotá: Kimpres.

American Educational Research Asociation. (2011). Code of Ethics. https://cdn.ymaws.com/wera.siteym.com/ resource/resmgr/a_general/aera.pdf

Alcántara, G. (2008). La definición de salud de la Organización Mundial de la Salud y la interdisciplinariedad. Revista Universitaria de Investigación, (1), 93-107.

Alva, L., \& Castillo, L. (2018). Espiritualidad y estilo de vida en estudiantes de Medicina Humana de la Universidad Peruana Unión. Revista Científica de Ciencias de la Salud, 11(1), 44-49.

Arango, O. \& Solano, O. (2016). La espiritualidad en Ignacio Ellacuría. Theologica Xaveriana, 66(181), 123-145. https://doi.org/10.11144/javeriana.tx66-181.eie

Azcuy, V. (2011). La espiritualidad como disciplina teológica: panorama histórico, consensos y perspectivas actuales. Revista Teología, 47(105), 251-280. 
Barrero, J., Clavijo, J., \& Gómez, N. (2011). Cuerpo sano y espiritual: prácticas de consumo y estilo de vida. Universitas Humanistica, 71(71), 175- 189. https://revistas.javeriana.edu.co/index.php/univhumanistica/ article/view/2197

Bauman, Z. (2013). La cultura en el mundo de la modernidad líquida. México: Fondo de Cultura Económica.

Bourdieu, P. (1998). La distinción. Criterio y bases sociales del gusto. Madrid: Taurus.

Bronfenbrenner, U. (1987). La ecología del desarrollo humano. Experimentos en entornos naturales y diseñados. Barcelona: Ediciones Paidós.

Castillo, J. (2008). Espiritualidad para insatisfechos. Madrid: Editorial Trotta.

Congreso de Colombia (2012). Ley Estatutaria 1581 de 2012. Diario Oficial No. 48.587. https://www.defensoria. gov.co/public/Normograma\%202013_html/Normas/Ley_1581_2012.pdf

Coral, C. \& Vargas, L. (2014). Estrategias que promueven estilos de vida alúdanlas. Revista U.D.C.A. Actualidady Divulgación Cientifica, 17(1), 35-43.

Cornejo, M., Martín, B., Esteso, C., \& Blázquez, M. (2019). El giro saludable: sacrificio, sanación, bienestar y su relación con la espiritualidad contemporánea. Athenea Digital-Revista de Pensamiento e Investigación Social, 19(2), 1-22. https://doi-org.ezproxy.javeriana.edu.co/10.5565/rev/athenea.2125.

Da Costa, B., Da Silva, K., Da Silva, J., Minatto, G., De Lima, L. \& Petroski, E. (2019). Sociodemographic, biological, and psychosocial correlates of light- and moderate-to-vigorous-intensity physical activity during school time, recesses, and physical education classes. Journal of Sport and Health Science, 8, 177-182.

Da Silva, A., Machado, W., Bellodi, A., Cunha, K. \& Enumo, S. (2018). Jovens insatisfeitos com a imagem corporal: estresse, autoestima e problemas alimentares. Psico-USF, 23 (3), 483-495.

De Souza, M., Pinzón, A. \& Zea, C. (2015). Analysis of the Lifestyle of Students of Pontificia Javeriana University Colombia. Medicine \& Science in Sports \& Exercise, 47 (5), 915.

De Souza, M., Zea, A., Rodríguez, G., \& Pinzón, A. (2017). Estilo de vida y factores socioeconómicos en estudiantes de electivas de actividad física y deporte de la Pontificia Universidad Javeriana. Análisis, 49(90), 229-243.

Delgado, M., (2015). Fundamentos de Psicología para Ciencias Sociales y de la Salud. Bogotá: Editorial Medica Panamericana.

Delors, J., Al Mufti, I., Amagi, I., Cameiro, R., Chung, F., Geremek, B. \& Nanzhao, Z. (1996). La Educación encierra un tesoro. Informe a la UNESCO de la Comisión Internacional sobre la educación para el siglo XXI. Madrid: Santillana; Ediciones UNESCO.

Dussel, E. (1974). El dualismo en la antropología de la cristiandad. Buenos Aires: Editorial Guadalupe.

Erikson, E. (1998). O Ciclo de vida completo. Porto Alegre: Artmed.

Espinosa, G. \& Carpio, L. (2015). Modelo dinámico ecológico de desarrollo humano de la deserción escolar en Aymaraes, Apurímac. Revista de Investigación en Psicología, 18 (2), 115-138.

Fernández, I., (2005). Construcción de una escala de actitudes tipo Likert. Madrid: Instituto Nacional de Seguridad e Higiene en el Trabajo.

Francisco. (2018). Constitución apostólica Veritatis gaudium, sobre las universidades y facultades eclesiásticas. Bogotá: San Pablo. 


\section{Espiritualidad y estilos de vida}

Gómez-Acosta, C. (2018). Factores psicológicos predictores de estilos de vida saludable. Revista de Salud Pública, 20(2), 155-162.

González-Carvajal, L. (2019). Espiritualidad cristiana en la ciudad. Revista de Espiritualidad, 78, 321-340.

Grüm, A. y Dufner, M. (2000). Una espiritualidad desde abajo. El diálogo con Dios desde el fondo de la persona. Madrid: Editorial Narcea.

Hadot, P. (2006). Ejercicios espirituales y filosofía. Madrid: Siruela.

Harari, Y. (2015). De animales a dioses. Breve historia de la humanidad. Bogotá: Géminis, S.A.S.

Harris, A. (2019). Report, finding our own way: Mental health and moving from school to further and higher education. London: Centre for Mental Health.

Hernández, R., Colorado, C. \& Lucio, P. (2014). Metodología de la investigación. México: Mc Graw Hill Education.

Hernández, R., \& Mendoza, C. (2018). Metodología de la investigación: las rutas cuantitativa, cualitativa y mixta. México: Mc Graw Hill Education.

Hurtado, A. S.J. (1941). ¿Es Chile un país católico? Santiago: Splendor.

Koenig, H. (2013). Is Religion good for your health. New York: Routledge.

Krmpotic, C. (2016). La espiritualidad como dimensión de la calidad de vida. Exploraciones conceptuales de una investigación en curso. Scripta Ethnologica, 38, 105-120.

Little, T. (2013a). The Oxford Handbook of Quantitative Methods, Volume1: Foundations. New York: Oxford University Press.

Little, T. (2013b). The Oxford Handbook of Quantitative Methods, Volume2: Statistical Analysis. New York: Oxford University Press.

López-Roldán, P. \& Fachelli, S. (2017). Metodología de la investigación social cuantitativa. Bellaterra: Universitat Autónoma de Barcelona.

Lucchetti, G., Lucchetti, A. L. G., Espinha, D. C. M., de Oliveira, L. R., Leite, J. R., \& Koenig, H. G. (2012). Spirituality and health in the curricula of medical schools in Brazil. BMC Medical Education, 12, 78.

Ministerio de Salud y Protección Social (2013). Resolución Número 008430 de 1993. https://urosario.edu.co/ Escuela-Medicina/Investigacion/Documentos-de-interes/Files/resolucion_008430_1993.pdf

Ministerio de Comercio, Industria y Turismo (2013). Decreto Número 1317 de 2013. http://wsp.presidencia.gov. co/Normativa/Decretos/2013/Documents/JUNIO/27/DECRETO\%201377\%20DEL\%2027\%20DE\%20 JUNIO\%20DE\%202013.pdf

Moscoso, D., Martin, M., Pedrajas, N. \& Sánchez, R. (2013). Sedentarismo activo. Ocio, actividad física y estilos de vida de la juventud española. Arch Med Deporte, 30(6), 341-437.

Nahas, M. (2000). Pentaculo de Bem-estar: Ejemplos de percepción e interpretación del perfil de estilos de vida. Revista Brasileira Actividade Física y Saude, 5(2), 34-35.

Nahas, M. (2013). Atividade física, saúde e qualidade de vida: conceitos e sugestôes para um estilo de vida ativo. Londrina: Midiograf.

Navas, C. \& Villegas, H. (2006). Espiritualidad y salud. Revista ciencias de la educación, 1 (27), 29-45. 
Organización Mundial de la Salud (2013). Plan de acción sobre salud mental 2013-2020. Ginebra: World Health Organization.

Organización Mundial de la Salud (1948). Constitución de la Organización Mundial de la Salud. https://www.who. int/es/about/who-we-are/constitution.

Pablo VI. (1965). Optatam totius, decreto sobre la formación sacerdotal. En Vaticano II. (pp. 526-554). Madrid: Biblioteca de Autores Cristianos.

Pablo VI. (1967). Constitución pastoral Gaudium et spes, sobre la iglesia en el mundo actual. En Vaticano II. (pp. 260-273). Madrid: Biblioteca de Autores Cristianos.

Palacio, C. (2015). La espiritualidad como medio de desarrollo humano. Revista Cuestiones Teológicas, 42(98), 459-481.

Papalia, D., Feldman, R. \& Martorell, G. (2012). Desarrollo Humano. Bogotá: McGraw-Hill Education.

Quiceno, J. \& Vinaccia, S. (2009). La salud en el marco de la psicología de la religión y la espiritualidad. Revista Diversitas - Perspectivas en Psicologia, 5(2), 321-336.

Quispe, W. (2018). Espiritualidad y estilo de vida en miembros de la IASD del distrito misionero Huancayo-Chilca de la Misión Andina Central 2017. [Trabajo de grado orientado a obtener el título de Licenciado en Teología, Universidad Peruana Unión]. https://repositorio.upeu.edu.pe/handle/UPEU/1225

Redacción Vida. (2017, 12 de abril). Colombia, entre los diez países más católicos del mundo. Diario El Tiempo. https://www.eltiempo.com/colombia/otras-ciudades/colombia-es-uno-de-los-diez-paises-mas-catolicos-delmundo-77648

Salgado, A. (2015). Formación universitaria en psicología de la religión y la espiritualidad: ¿¿Necesidad o Utopía? Revista Digital de Investigación en Docencia Universitaria, 2, 89-103. https://dialnet.unirioja.es/servlet/ articulo?codigo $=5282389$

Sánchez, B. (2008). Comparación entre el bienestar espiritual de pacientes del Programa de Enfermería Cardiovascular y el de personas aparentemente sanas. Avances en Enfermería, 36(1), 65-74.

Shelton, L. (2019). The Bronfenbrenner primer, a guide to develecology. New York: Routledge.

Silva, H. (1977). La espiritualidad apostólica hoy. Theologica Xaveriana, (45). https://revistas.javeriana.edu.co/index. $\mathrm{php} /$ teoxaveriana/article/view/26852

Tamayo, J. (2007). Espiritualidad y respeto de la diversidad. Conferencia pronunciada en el II Foro Mundial de Teología y Liberación. http://www.donesesglesia.cat/documentos/Tamayo-Nairobi.pdf

Ukaski, M. (2008). Espiritualidad Sanante. Salud y espiritualidad en Anselm Grün. Revista Teología, 45 (27), 633-653.

Uresti, M., Ramírez, J. \& Caballero-Rico, F. (2011). Salud y espiritualidad: no solo de pan vive el hombre. CienciaUAT, $6(2), 48-54$.

Zea, A., León-Ariza, H., Botero-Rosas, D., Afanador-Castañeda, H. \& Pinzón-Bravo, L. (2014). Factores de riesgo cardiovascular y su relación con la composición corporal en estudiantes universitarios. Revista Salud Pública, 16(4), 505-515. 